\title{
Exciplexes or ground state complexes of (dibenzoylmethanato)boron difluoride and benzene derivatives? A study of their optical properties revisited via liquid state investigations and structure calculations.
}

\section{Thanh-Toan Truong ${ }^{ \pm}$, Valérie Brenner, Gilles Ledoux ${ }^{\dagger}$ and Thu-Hoa Tran-Thi*}

CEA-Saclay, DSM/DRECAM/SPAM/Laboratoire Francis Perrin, URA CEA-CNRS 2453, 91191 Gif-sur-Yvette Cedex, France

tpresently in Laboratoire de Physico Chimie des Matériaux Luminescents, Université Claude Bernard - Lyon I - CNRS UMR 5620, 69622 Villeurbanne Cedex, France

${ }^{ \pm}$presently in CEA-Saclay, DRT/LITEN/DSEN/GENEC/Laboratoire Cellules et Composants, 91191 Gif-sur-Yvette Cedex, France

\section{Abstract}

(Dibenzoylmethanato)boron difluoride (DBMBF2) was found to form with benzene (B) and its methylated derivatives (MB) in cyclohexane two types of ground state complexes. The first complexes with low stoichiometries 2:1, 1:1 and 1:2 do not fluoresce when they are excited. On the other hand, the ground state complexes with high stoichiometry, DBMBF2:(B) $)_{n}$ or DBMBF2:(MB) $($ with $n>>2$ ), exhibit a strong fluorescence in their excited states. These findings differ from the previous works, where the strongly fluorescing complexes have been argued to be the 1:1 and 1:2 exciplexes, complexes of the singlet excited state of DBMBF2 with one or two B or MB molecules. These differences are discussed in terms of the solute-solute and solute-solvent interactions when DBMBF2 and $\mathrm{MB}$ are solutes in cyclohexane or when $\mathrm{MB}$ is a co-solvent of cyclohexane in binary mixtures of DBMBF2. We also argue that the use of well-suited analytical methods is important for the determination of the nature of the various complexes. Furthermore, to understand the nature of the interactions between benzene and DBMBF2 molecules, we attempt to predict the sites of interaction between DBMBF2 and benzene molecules by determining theoretically the structure of the 1:1 complex.

\section{$1 \quad$ Introduction}

In order to elaborate BTXs (benzene, toluene and xylenes) sensors for measurements of air quality [1], we have been seeking fluorescent probe molecules able to interact with monocyclic aromatic compounds. Thus, (dibenzoylmethanato)boron difluoride (DBMBF2) appeared to be a good candidate, since previous works reported its ability to form highly fluorescent exciplexes with monocyclic aromatic compounds in cyclohexane [2-4] or acetonitrile [5] solutions. Moreover, these exciplexes were found to display fluorescence spectra which shift to the red with increasing number of methyl groups on the benzene ring $[2,3]$. One could make use of these particularly interesting properties to obtain a good selectivity for a sensor. For this particular application, the DBMBF2 molecules were incorporated under vacuum into a porous matrix. Upon exposure of the matrix to gaseous benzene or toluene diluted in nitrogen, we were expecting to observe the formation of the fluorescent 1:1 and 1:2 exciplexes. In contrast to our expectation, we observe small alteration of the DBMBF2 absorption bands and the extinction of the fluorescence of DBMBF2 monomer, but no new fluorescence. These intriguing results prompted us to look more carefully at the previous data. In particular, we noted that, in previous works, very high concentrations of the aromatic compounds were needed to be able to detect the exciplexes. In 
cyclohexane solutions, the minimum amount of benzene compound to be added to the solution in order to detect the exciplex fluorescence is $0.094 \mathrm{~mol} . \mathrm{L}^{-1}$ and corresponds to a molar ratio aromatic:DBMBF2 of 9400 [3]. With such concentrations, the aromatic compound becomes a co-solvent of cyclohexane and the properties of DBMBF2 correspond to those of DBMBF2 in binary mixtures. These considerations had prompted us to revisit these systems with a new eye. In a first step, we want to reproduce the previous experiments with DBMBF2 in binary mixtures of cyclohexane and benzene (or toluene). Then, in a second step, we will study the interactions between DBMBF2 and benzene (or toluene) molecules, when both are solutes in cyclohexane. To this end, we will make use of the Job method [6] and its extension to fluorimetric titration [7], two analytical methods suited to low concentrations of reactants, which will enable the determination of ground state complexes of DBMBF2 with the aromatic compound in cyclohexane. From the Job plots in absorption and from the study of the variation of the total fluorescence of the mixtures, one can obtain the stoichiometry of the-complexes and their association constant. Moreover, we also attempt to predict the sites of interaction between DBMBF2 and benzene by determining the geometry of the ground state 1:1 complex in vacuum, using semi-empirical quantum chemistry calculations.

The outline of the remainder of this paper is as follows. After the experimental section, we report and discuss the results obtained with benzene and toluene, using two analytical methods of titration of DBMBF2 with the aromatic compound, a first one which reproduces the previous experimental conditions of the literature (high concentration of the aromatic compound) and the Job method from which are extracted the stoichiometry of the complexes and their association constants. We then discuss the structure of the three isomers of the 1:1 complex of DBMBF2 with benzene predicted from the calculations and finally conclude on the nature of non-fluorescent and fluorescent complexes.

\section{Experimental section \\ 2.1. Chemicals}

(Dibenzoylmethanato)boron difluoride was prepared according to the procedure described by Brown and Bladon [8] and re-crystallized three times from ether giving a yellow powder: mp: $194-196{ }^{\circ} \mathrm{C}$. The compound was analyzed via proton NMR and ${ }^{19} \mathrm{~F}$ NMR in $\mathrm{CDCl}_{3}$. All chemicals are of spectroscopic or analytical grade and are used without further purification. Benzene free cyclohexane $(\geq 99.5 \%)$ for UV spectroscopy is from Fluka and toluene of spectrophotometric grade $(99.5 \%)$ is from Aldrich. Ethanol (EtOH) of Uvasol grade is from Merck.

\subsection{Titration methods}

Two titration methods were used depending on the concentration range of benzene and toluene. When the aromatic hydrocarbon concentration is high enough to be considered as a co-solvent of cyclohexane, two mother mixtures of DBMBF2 $10^{-5} \mathrm{mol.L}^{-1}$ in cyclohexane and DBMBF2 $10^{-5} \mathrm{~mol} . \mathrm{L}^{-1}$ in benzene (or toluene) were prepared. Various mixtures were then prepared from these two mother solutions to provide various concentrations of the aromatic, while the DBMBF2 concentration was always kept constant and equal to $10^{-5} \mathrm{~mol} . \mathrm{L}^{-1}$. On the other hand, when the analytical method of Job [6,7] was used, two solutions of DBMBF2 in cyclohexane and benzene (or toluene) in cyclohexane with a ratio of concentrations [Aromatic] $/[\mathrm{DBMBF} 2]>10$, at least, were prepared. Addition of small aliquots of the benzene solution in cyclohexane then gave new mixtures where 
DBMBF2 and the aromatic hydrocarbon remained solutes over the whole range of concentration of the added compound.

\subsection{Steady-state absorption and fluorescence and time-resolved fluorescence.}

The UV and visible spectra were recorded with a Perkin-Elmer Lambda 900 spectrometer. The extinction coefficient values of DBMBF2 in the various solvents are collected in Table 1. Fluorescence spectra of DBMBF2 in the binary mixtures were recorded with a $1 \mathrm{~cm}$ path length fluorimeter cell over the UV-visible domain. The excitation source of the SPEX-Fluorolog fluorimeter is a $450 \mathrm{~W}$ XBO Xenon lamp. When the DBMBF2 concentration is higher than $10^{-5}$ mol. $\mathrm{L}^{-1}$, optical cells with 2 or $1 \mathrm{~mm}$ path length were used to collect in a front-face configuration the fluorescence spectra of the solutions. The fluorescence spectra were corrected for the monochromator and photomultiplier response over the 250-750 nm range. Similarly, the excitation spectra of the mixtures were collected and compared with the absorption spectra of the same mixtures. DBMBF2 fluorescence quantum yield, $\Phi_{\mathrm{f}}$, in cyclohexane, benzene and toluene were determined (see Table 1) with very dilute solutions of DBMBF2 using as standard the Quinine sulfate dihydrate [9].

Table 1: Optical properties of DBMBF2 in various solvents

\begin{tabular}{|c|c|c|c|}
\hline & Cyclohexane & Benzene & Toluene \\
\hline \multicolumn{4}{|l|}{ Abs } \\
\hline$\lambda / n m$ & $\begin{array}{l}\lambda_{1}=269 \pm 1 \\
\lambda_{2}=360 \pm 1\end{array}$ & $\lambda=366 \pm 1$ & $\lambda=367 \pm 1$ \\
\hline$\varepsilon / L . \mathrm{mol}^{-1} . \mathrm{cm}^{-1}$ & $\begin{array}{l}\varepsilon_{1}=8800 \pm 600 \\
\varepsilon_{2}=38700 \pm 2900\end{array}$ & $\varepsilon=33250 \pm 1550$ & $\varepsilon=30450 \pm 1500$ \\
\hline \multicolumn{4}{|l|}{ Fluo } \\
\hline$\lambda_{\max } / n m$ & $\begin{array}{l}\lambda_{\mathrm{f} 1}=389 \pm 1 \\
\lambda_{\mathrm{f} 2}=409 \pm 1\end{array}$ & $\lambda_{\mathrm{f} 1}=425 \pm 1$ & $\lambda_{f}=440 \pm 1$ \\
\hline$\Phi_{\text {fluo }} /$ a.u. & $\begin{array}{l}{ }^{\mathrm{a}} 0.046 \\
{ }^{\mathrm{b}} 0.057 \pm 0.005\end{array}$ & $\begin{array}{l}{ }^{\mathrm{a}} 0.44 \\
{ }^{\mathrm{b}} 0.55 \pm 0.05\end{array}$ & $\begin{array}{l}{ }^{\mathrm{a}} 0.68 \\
{ }^{\mathrm{b}} 0.68 \pm 0.07\end{array}$ \\
\hline$\tau_{S} / n s$ & $\begin{array}{l}{ }^{\mathrm{a}} 0.14 \\
{ }^{\mathrm{b}} 0.17 \pm 0.03\end{array}$ & ${ }^{\mathrm{a}} 2.1$ & ${ }^{\mathrm{a}} 4.1$ \\
\hline
\end{tabular}

\subsection{Calculations of the potential energy surface of the DBMBF2:benzene 1:1 complex.}

Prior to the investigation of the complexation sites of the DBMBF2 via calculations of the potential-energy surface of the DBMBF2:benzene system, the geometries of the most stable isolated DBMBF2 and benzene were first investigated. For DBMBF2, the crystal structure known from the literature [10] was compared with the geometries of the different isomers that we generated using the semi-empirical quantum chemistry method [11] by varying the spatial arrangements of some chemical groups, namely the BF2 group and the aromatics cycles. From this procedure, various geometries with local minima were obtained among which the two lowest were kept and optimized at the B3LYP/6-31G** level [12] using the pseudo-spectral method of Jaguar 4.1 package. This procedure led to a single geometry in agreement with the X-rays structure [10], corresponding to the symmetrical isomer, which was finally refined at the MP2/6-31G** level using Gaussian 98 [13]. The geometry of benzene was optimised first at the B3LYP/CC-pVDZ [14] level using the pseudo-spectral method of Jaguar 4.1 [15] and then refined at the MP2/6-311G (2d, 2p) [16] level using Gaussian 98 [13]. 
The potential assumes intramolecular geometries to be frozen and the number of degrees of freedom were six (three translations and three rotations, Euler angles). Based on the exchange perturbation theory, it contains electrostatic, polarization, repulsion and dispersion contributions. The electrostatic interaction was calculated as a sum of all two-body multipole-multipole interactions. The multipolar distribution for each molecule (a charge, a dipole, a quadrupole on each atom and bond center) was derived from the wave function obtained by an ab initio calculation at the MP2/311G** [16] level using Gaussian 98 [13] and according to the method of Vigné Maeder et al [17]. The dipole polarization interaction results from a self-consistent iterative procedure during which additive site polarizabilities on each molecule respond to the electric field created by the (permanent and induced) moments on all other molecules. The dispersion and repulsion terms were calculated as the sum of atom-atom terms. The repulsion term is expressed by an exponential form. The dispersion terms contain terms up to $\mathrm{C}_{10} / \mathrm{R}^{10}$ as well as an exchange contribution. As the repulsion and dispersion parameters of the boron atom and the polarizabilities of the $\mathrm{B}-\mathrm{O}$ and $\mathrm{B}-\mathrm{F}$ bonds were not available, they were respectively replaced by the parameters of the carbon and the polarizabilities of the C-O and C-F bonds.

The potential-energy surface of the system DBMBF2:benzene was then investigated using a global optimisation method [18] based on the simulated annealing method [19]. The exploration of the potential-energy surface was carried out in two steps. First, a random search on the surface is performed using the Metropolis algorithm [20] associated with a controlled temperature parameter, which is decreased according to an annealing schedule. Secondly, the configurations obtained from this exploration are sorted out and finally the resulting configurations are optimized with a local minimization method, a quasi-Newton method-type, the BFGS method [21]. Among the different configurations obtained, we select the three lowest potential energy configurations, the other configurations lying at significantly higher energy.

\section{$3 \quad$ Results and discussion}

\subsection{Binary mixtures of benzene and cyclohexane containing DBMBF2}

\subsubsection{Studies in absorption}

We first try to reproduce the literature data with binary mixtures. In these mixtures, the lowest concentration of benzene $(0.094 \mathrm{M})$ corresponds to a benzene/DBMBF2 molar ratio of 9400 and the molar fraction of the two co-solvents is varied from 0 to 1 . The spectral evolution of DBMBF2 in the binary mixtures of benzene and cyclohexane over the UV domain is shown in Figure 1. As the concentration of benzene increases from 0.088 to 0.794 mol. $\mathrm{L}^{-1}$, the absorption maximum remains unchanged, while at higher concentrations up to 11.25 mol. $\mathrm{L}^{-1}$ (pure benzene), a continuous red shift of the absorption band is observed for the lowest transition of DBMBF2. Moreover, two isobestic points at 318 and $371 \mathrm{~nm}$ clearly appear during the titration over the whole concentration range of benzene. These observations are different from the literature data which mentioned no significant change in the absorption bands and no presence of any isobestic point [5]. 

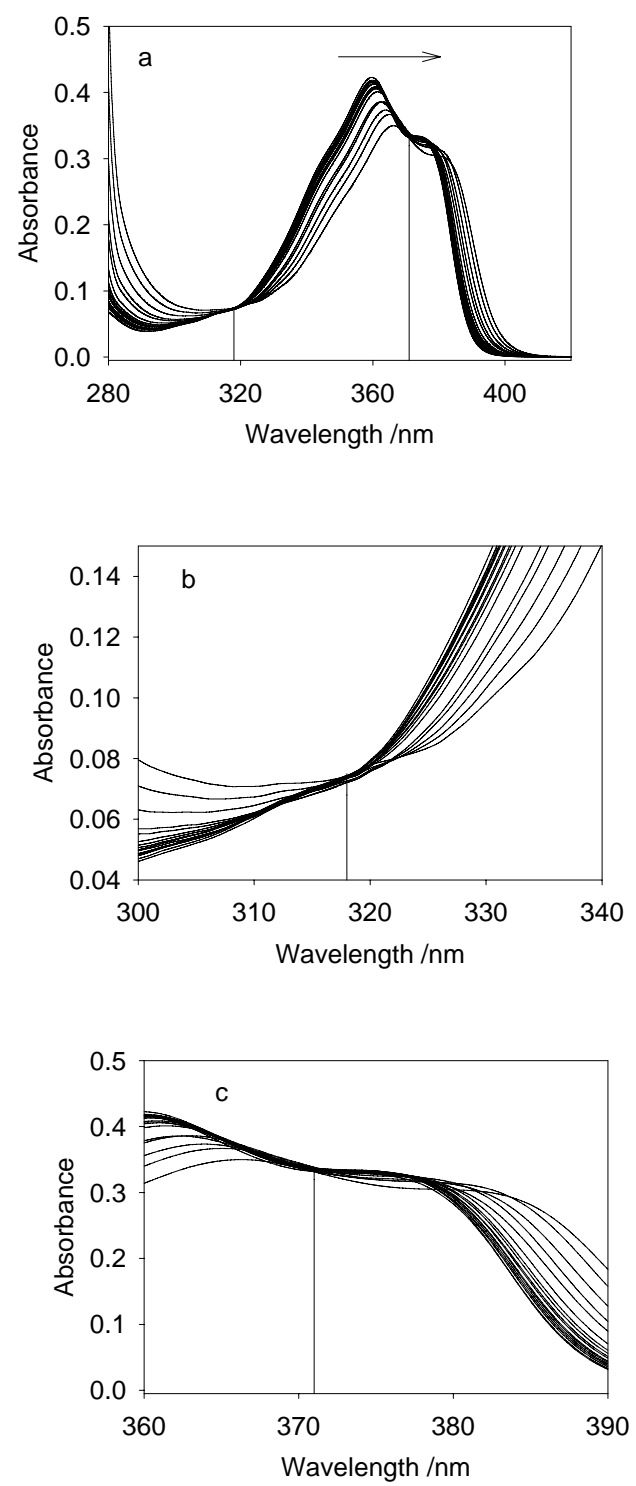

Fig.1 (a): Spectral evolution of DBMBF2 $10^{-5}$ mol. $\mathrm{L}^{-1}$ in cyclohexane containing various benzene concentrations (left to right): $0,0.088,0.200,0.300,0.395,0.494,0.596,0.794,0.996,1.194$, $1.492,1.994,2.981,3.980,5.978,7.909,11.252$ (pure benzene) mol.L $\mathrm{L}^{-1}$. (b): Zoom on the isobestic point at $318 \pm 1 \mathrm{~nm}, \operatorname{Abs}(318 \mathrm{~nm})=0.074 \pm 0.002$. (c): Zoom on the second isobestic point at $371 \pm 2 \mathrm{~nm}, \operatorname{Abs}(371 \mathrm{~nm})=0.335 \pm 0.004$

In the present case, the presence of the isobestic points argues for the existence of the equilibrium between two ground state species in solution, the DBMBF2 monomer and a complex formed with benzene molecules. On the other hand, the gradual red-shift of the position of the maximum of absorption, $\lambda_{\max }$, of the lowest $S_{0} \rightarrow S_{1}$ transition of DBMBF2 as a function of the molar fraction of benzene could be due to a gradual change of the complex solvation shell induced by the replacement of cyclohexane by benzene molecules (Fig. 2). As a matter of fact, the red shift starts to be significant when the mole fraction of benzene becomes bigger than 0.1 . This hypothesis will be strengthened with the coming fluorescence data. 


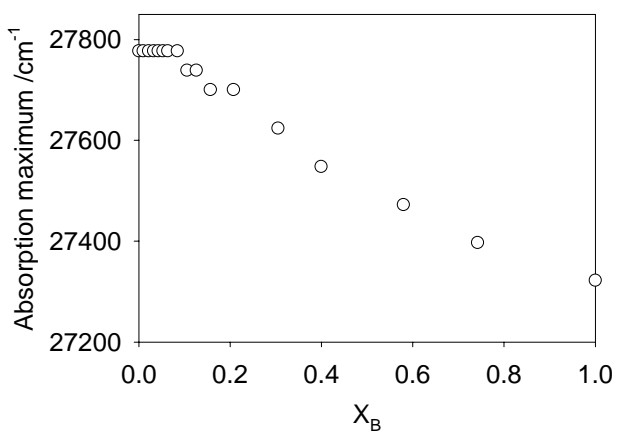

Fig.2 Evolution of the position of the maximum of absorption of the $\mathrm{S}_{0} \rightarrow \mathrm{S}_{1}$ transition of DBMBF2 as a function of the molar fraction of benzene $\mathrm{X}_{\mathrm{B}}$. See Table 2 for the precise values of max. and $\mathrm{X}_{\mathrm{B}}$.

\subsubsection{Fluorescence studies}

By exciting DBMBF2 in the binary mixtures of benzene and cyclohexane at the isobestic wavelength $(318 \mathrm{~nm})$, we can study the properties of the excited states of the two species in equilibrium in the ground state. We can note, similarly to the literature data [2,3], a red shift of the maximum and broadening of the fluorescence band of DBMBF2 with increasing benzene concentration (see Fig.3). Similarly to the spectral variations in absorption, the red shift of the fluorescence band becomes significant when the molar fraction of benzene becomes higher than 0.08 (see Fig.4). On the other hand, we can observe for the first time the existence of an isoemissive point at $386 \mathrm{~nm}$, which argues for the presence of an equilibrium between two fluorescing species (Fig. 3).

To demonstrate that the equilibrium in the excited state reflects the one in the ground state, one should collect the excitation spectra at the same fluorescence wavelength, $386 \mathrm{~nm}$, corresponding to the isoemissive point. However, in such a case, the excitation spectra would be limited to the 250-365 $\mathrm{nm}$ domain. Therefore, we collect the excitation spectra at the same fluorescence wavelength, $440 \mathrm{~nm}$. The excitation spectra normalized to the fluorescence intensity at $440 \mathrm{~nm}$, display two isobestic points at $303 \pm 2$ and $378 \pm 2 \mathrm{~nm}$. When comparing the absorption and excitation spectra, we can note that:

- The spectral evolution of the absorption and excitation spectra is very similar. A slight difference is observed for the ratio of the absorbances at 360 and $380 \mathrm{~nm}$. This ratio, which is high for DBMBF2 in pure cyclohexane and is less important in pure benzene, could reflect the proximate environment or salvation shell of DBMBF2 in the mixtures.

- The positions of the two isobestic points (303 and $378 \mathrm{~nm})$ are different from that of the absorption spectra (318 and $371 \mathrm{~nm})$. This difference reflects the weight of the two fluorescing species in equilibrium, which contribute to the fluorescence intensity at $440 \mathrm{~nm}$, and is consistent with the difference of shape dicussed above. Note that we would have obtained the same isobestic points at 318 and $371 \mathrm{~nm}$ for the excitation spectra if it were possible to collect the entire excitation spectra at the isoemissive wavelength, $386 \mathrm{~nm}$.

These results strongly support the hypothesis of the existence of the equilibrium between two species formed in the ground state and which both fluoresce when they are excited. 

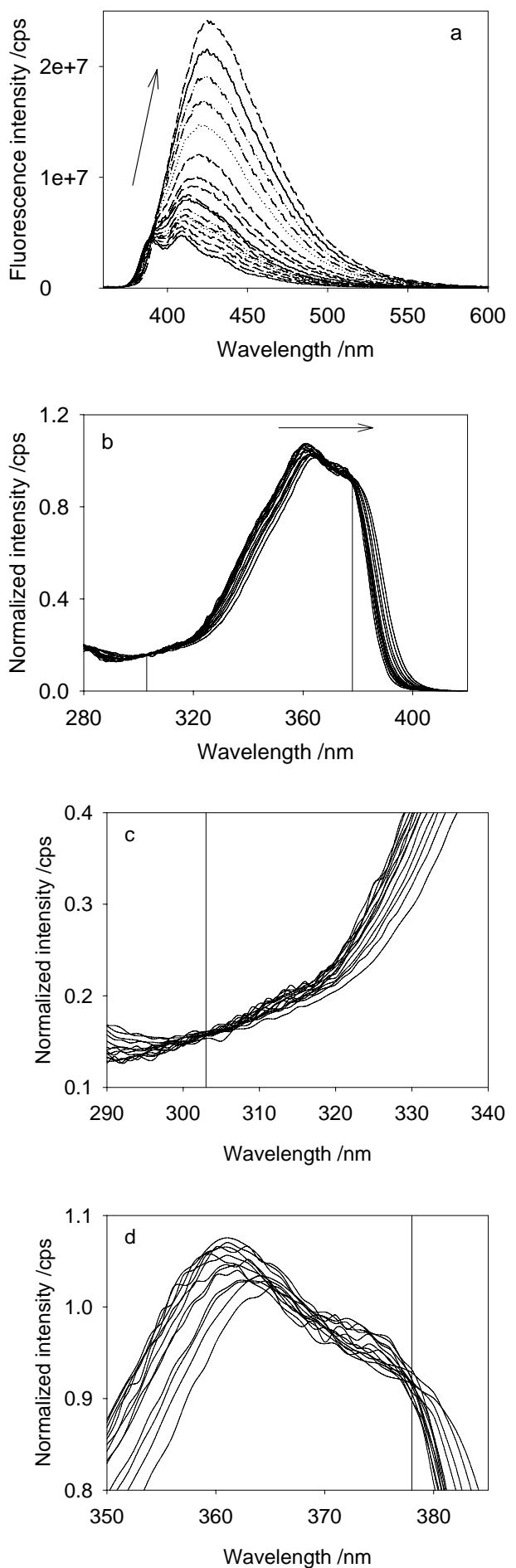

Fig.3 (a): Fluorescence spectra of DBMBF2 $10^{-5}$ mol. $\mathrm{L}^{-1}$ in the binary mixtures of benzene and cyclohexane. Benzene concentrations (left to right): 0, 0.088, 0.200, 0.300, 0.395, 0.494, 0.596, $0.794,0.996,1.194,1.492,1.994,2.981,3.980,5.978,7.909,11.252$ (pure benzene) mol.L $L^{-1}$.

(b): Excitation spectra obtained at $\lambda_{\mathrm{em}}=440 \mathrm{~nm}$ and normalized to the fluorescence intensity.

(c): Zoom on the isobestic point of the excitation spectra at $303 \pm 2 \mathrm{~nm}$. (d): Zoom on the second isobestic point at $378 \pm 2 \mathrm{~nm}$. 
From data of figures 2 and 4, we calculate the Stokes shift value corresponding to the lowest transition of DBMBF2 for each binary mixture (see Table 2). The Stokes shift dramatically increases when the benzene molar fraction becomes higher than 0.08 and remains approximately constant for higher values up to 1 . The observation of a jump in the Stokes shift value and broadening of the fluorescence band is in favor of the formation of a charge transfer complex, CT, of DBMBF2 and benzene. Over a benzene molar fraction of 0.1 , the red shift of the fluorescence band could be attributed to the change in the solvation shell of the charge transfer complex, due to the replacement of cyclohexane by benzene molecules. The stoichiometry of this complex is presently questioned because when the molar fraction of benzene is equal to 0.08 , there are actually 76000 molecules of benzene and 860000 molecules of cyclohexane for one DBMBF2.

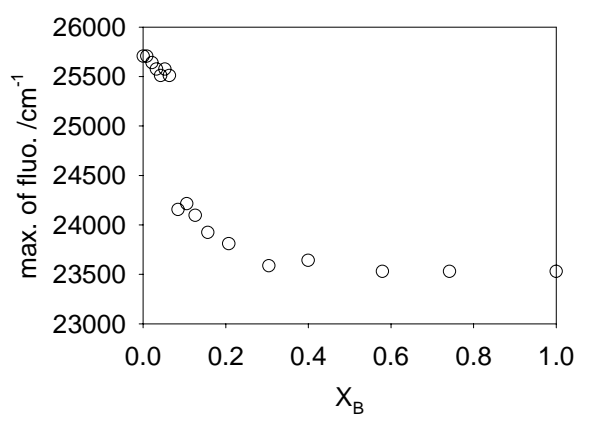

Fig.4 Evolution of the position of the maximum of fluorescence of DBMBF2 $10^{-5} \mathrm{~mol} \cdot \mathrm{L}^{-1}$ in the binary mixtures of benzene and cyclohexane as a function of the molar fraction of benzene $\left(\mathrm{X}_{\mathrm{B}}\right)$. see Table 2 for the precise values of max. and $\mathrm{X}_{\mathrm{B}}$.

Table 2: Absorption, fluorescence maxima and Stokes shift of DBMBF2 $10^{-5} \mathrm{~mol}^{-\mathrm{L}^{-1}}$ in benzenecyclohexane binary mixtures

\begin{tabular}{|c|c|c|c|c|c|}
\hline [B] mol. $\mathrm{L}^{-1}$ & [cHex] mol.L L $^{-1}$ & $X_{B}$ & Abs & Fluo & Stokes shift \\
\hline 0 & 9.256 & $\overline{00}$ & 27778 & 25707 & 2071 \\
\hline 0.088 & 9.183 & 0.009 & 27778 & 25707 & 2071 \\
\hline 0.200 & 9.091 & 0.022 & 27778 & 25641 & 2137 \\
\hline 0.300 & 9.010 & 0.032 & 27778 & 25575 & 2203 \\
\hline 0.395 & 8.931 & 0.042 & 27778 & 25510 & 2268 \\
\hline 0.494 & 8.850 & 0.053 & 27778 & 25575 & 2203 \\
\hline 0.596 & 8.766 & 0.064 & 27778 & 25510 & 2268 \\
\hline 0.794 & 8.603 & 0.085 & 27778 & 24155 & 3623 \\
\hline 0.9961 & 8.437 & 0.106 & 27739 & 24213 & 3526 \\
\hline 1.194 & 8.274 & 0.126 & 27739 & 24096 & 3643 \\
\hline 1.492 & 8.029 & 0.157 & 27701 & 23923 & 3778 \\
\hline 1.994 & 7.616 & 0.207 & 27701 & 23810 & 3891 \\
\hline 2.981 & 6.803 & 0.305 & 27624 & 23585 & 4039 \\
\hline 3.980 & 5.982 & 0.400 & 27548 & 23641 & 3907 \\
\hline 5.978 & 4.339 & 0.579 & 27472 & 23529 & 3943 \\
\hline 7.909 & 2.750 & 0.742 & 27397 & 23529 & 3868 \\
\hline 11.252 & 0 & 1 & 27322 & 23529 & 3793 \\
\hline
\end{tabular}

Abs, Fluo and Stokes shift units are expressed in $\mathrm{cm}^{-1}, \mathrm{X}_{\mathrm{B}}$ is the benzene molar fraction

The present findings differ from previous data reported by Chow et al. on many points:

For the binary mixtures of cyclohexane and benzene containing DBMBF2, these authors did not observe any isobestic point in the spectral evolution of DBMBF2 and concluded to the 
absence of ground state complex in solution. They however mentioned that bright yellow crystals of DBMBF2 could become colorless when in contact with benzene vapor and recover their yellow color when exposed to air [4] and attributed this effect to the formation of a ground state complex of the two reactants, which is unstable towards oxygen. On the other hand, the same authors reported the existence of ground state complexes between DBMBF2 and various electron-rich or electron-donor compounds such as quadricyclene, trimetylamine, cyclohexadiene or trans-anethole (1-methoxy-4-propenyle benzene) in acetonitrile [4]. With trans-anethole, the absorption spectrum of DBMBF2 develops a new absorption which extends beyond the longest wavelength edge from 420 to $520 \mathrm{~nm}$ [4]. This new absorption also appears, though in a lesser extent over $420-435 \mathrm{~nm}\left(\sim 820 \mathrm{~cm}^{-1}\right)$, in ternary systems with DBMBF2, $p$-xylene and ethylene diamine (EDTA) in acetonitrile [22] and was assigned to a ground state charge transfer complex of DBMBF2:p-xylene stabilized in the presence of EDTA. In this last case, the red extension of the DBMBF2 absorption band appears to us very similar to the one we presently observe over $390-400 \mathrm{~nm}\left(\sim 640 \mathrm{~cm}^{-1}\right)$ for DBMBF2 in cyclohexane upon addition of benzene. If we applied the same reasoning than these authors, our present observations would support the existence of a ground state CT complex between DBMBF2 and benzene.

Because they did not observe the presence of any isobestic point in the absorption bands of DBMBF2 in the binary mixtures, to collect the fluorescence, Chow et al. [2,3] have excited DBMBF2 either at 389 [3] or $366 \mathrm{~nm}$ [4], two wavelengths close to the position of the maximum of the lowest transition of the DBMBF2. As it can be seen from Figure 1, the intensity of this transition continuously varies upon increasing amount of benzene in the mixtures and therefore the number of photons absorbed by the solutions was not kept constant. This effect could be important in particular at $389 \mathrm{~nm}$ in the red tail of the absorption band as the absorbance varies from 0.044 to 0.208 (Fig.1). The total fluorescence area measured by these authors fluctuates with the absorbance change of the mixture and can not be simply normalized. Therefore the validity of the model involving the existence of two charge transfer exciplexes with 1:1 (DBMBF2:benzene) and 1:2 (DBMBF2:(benzene) $\left.)_{2}\right)$ stoichiometries [3] is questioned.

In the present work, the similarity between the absorption spectra of the binary mixtures and the corresponding excitation spectra recorded from fluorescence measurements at $440 \mathrm{~nm}$ (compare Fig. 1(a) and Fig.3b), strongly supports the existence of ground state complexes. Both series of absorption and excitation spectra display a set of two isobestic points at 318 and 371 and 303 and $378 \mathrm{~nm}$, respectively. Note again that the difference of the position of the set of isobestic points is due to the fact that the excitation spectra were collected at $440 \mathrm{~nm}$ and not at the isoemissive point, $386 \mathrm{~nm}$. These results clearly indicate that the fluorescence spectra correspond to the two emitting species which are in equilibrium in the ground state. Such isobestic points would not be observed if the broad fluorescence corresponds to exciplexes. However, at this point, the nature of the fluorescent complex is not yet elucidated and further experiments are needed.

\subsection{Determination of the stoichiometry of the complexes in cylohexane solutions}

\subsubsection{The Job method}

To determine the stoichiometry of the complexes, we use the analytical method of Job [6] and cyclohexane solutions in which the two reactants, DBMBF2 and benzene (or toluene) are solutes. The method of Job [6] and its extension to fluorimetric titration [7] have been commonly used to experimentally determine the stoichiometry of the ground state complexes 
and were successfully used for tetrapyrrole systems [7]. It relies on the fact that the absorbance of a mixture of chromophores A (=DBMBF2) and B (=benzene or toluene) which do not interact with each other is the sum of the absorbances of each chromophore.

do $^{\text {ideal }}=\varepsilon_{\mathrm{A}} \mathrm{C}_{\mathrm{A}} 1+\varepsilon_{\mathrm{B}} \mathrm{C}_{\mathrm{B}} 1$

where $\varepsilon_{\mathrm{i}}$ is the molar absorptivity of the compound $\mathrm{i}$ at a given wavelength, $\mathrm{C}_{\mathrm{i}}$ the concentration of compound $\mathrm{i}$ and 1 the optical pathlength. do ${ }^{\text {ideal }}$ can also be expressed as a function of the molar fraction, $\mathrm{x}$, of compound $\mathrm{B}$ :

$\mathrm{do}^{\text {ideal }}=\mathrm{C}_{\mathrm{T}} 1\left[\varepsilon_{\mathrm{A}}(1-\mathrm{x})+\varepsilon_{\mathrm{B}} \mathrm{x}\right]$

with $\mathrm{x}=\mathrm{C}_{\mathrm{B}} / \mathrm{C}_{\mathrm{T}}$ and $\mathrm{C}_{\mathrm{T}}$ being the total concentration of compounds $\mathrm{A}$ and $\mathrm{B}$.

Conversely, if $\mathrm{A}$ and $\mathrm{B}$ react together to form a complex $\mathrm{AB}$, the absorbance do ${ }^{\text {read }}$ will differ from do ${ }^{\text {ideal }}$. Departures from the additivity, $\mathrm{F}(\mathrm{x})$, as the composition of the mixture is continuously varied, can be calculated:

$F(x)=\frac{d o^{\text {read }}}{C_{T} l}-(1-x) \varepsilon_{A}-x \varepsilon_{B}$

The Job plot corresponds to the plot of $\mathrm{F}(\mathrm{x})$ as a function of $\mathrm{x}$. When a ground state complex is formed, its stoichiometry can be deduced from the composition at which the deviation from additivity, $\mathrm{F}(\mathrm{x})$, is a maximum.

For fluorimetric titration, two main hypotheses are required: i) the two chromophores, $\mathrm{A}=\mathrm{DBMBF} 2$ and $\mathrm{B}=$ benzene (or toluene), can fluoresce but their concentration is low enough to prevent from long distance quenching; ii) the ground state complex $\mathrm{AB}$ formed is not fluorescent or displays a very different fluorescence spectrum.

$\mathrm{A}+\mathrm{B} \Leftrightarrow \mathrm{AB}$

The intensity of fluorescence (or fluorescence area) of a species i may be written as $\mathrm{F}_{\mathrm{i}}[\mathrm{i}]$, where [i] is the concentration of fluorophore $\mathrm{i}$ and $\mathrm{F}_{\mathrm{i}}$ a proportionality factor which depends on the quantum yield of fluorescence and on the excitation wavelength. Consider an initial solution of $\mathrm{A}$ at concentration $\mathrm{C}$, to which $\mathrm{n}$ equivalents of $\mathrm{B}$ have been added. As long as the value of $\mathrm{n}$ is not close to unity, the above reaction is completely displaced to the right and the formation of the complex $\mathrm{AB}$ may be considered quantitative. The overall fluorescence intensity (or area) from the solution may be written as:

For $\mathrm{n}<1: \quad \mathrm{I}_{1}(\mathrm{n})=\mathrm{C}\left[\left(\mathrm{F}_{\mathrm{AB}}-\mathrm{F}_{\mathrm{A}}\right) \mathrm{n}+\mathrm{F}_{\mathrm{A}}\right]$

For $\mathrm{n}>1: \mathrm{I}_{2}(\mathrm{n})=\mathrm{C}\left[\mathrm{F}_{\mathrm{AB}}+(\mathrm{n}-1) \mathrm{F}_{\mathrm{B}}\right]$

In a first approximation, the variation of the total fluorescence intensity as a function of $\mathrm{n}$ will consist in two linear regimes which intersect at $n=1$.

The choice of the excitation wavelength is very important when the concentrations of the reactants and product continuously vary during the titration. One needs to keep a constant number of absorbed photons during the whole titration. The ideal case is to excite the mixtures at a wavelength for which the absorbance of the mixtures remains practically constant or constant such as an isobestic point.

For both analytical methods in absorption and fluorescence, the two reactants, DBMBF2 and benzene (or toluene), must be kept at low concentration in the same solvent, cyclohexane. 


\subsubsection{Application to mixtures of DBMBF2 and benzene (or toluene) in cyclohexane}

Two experiments were performed: the first one corresponds to the titration of a DBMBF2 solution $\left(10^{-5}\right.$ mol. $\left.\mathrm{L}^{-1}\right)$ with a benzene solution $\left(1.810^{-3} \mathrm{~mol} . \mathrm{L}^{-1}\right)$ in cyclohexane. To increase the probability of complexation, the second titration was performed with a more concentrated solution of DBMBF2 $\left(10^{-4} \mathrm{~mol} \cdot \mathrm{L}^{-1}\right)$.

Figure 5 displays the spectral variation of DBMBF2 $10^{-5}$ mol. $\mathrm{L}^{-1}$ in cyclohexane upon addition of small aliquots of a solution of benzene $1.810^{-3} \mathrm{~mol}^{-1}$ in cyclohexane. A decrease of the absorbances centered at 360 and $269 \mathrm{~nm}$ corresponding to DBMBF2 monomer concomitant with the increase of the absorbance over 200-264 $\mathrm{nm}$ and the presence of an isobestic point at $264 \mathrm{~nm}$ argue for the existence of an equilibrium at least between two absorbing species. The plots of Job diagrams at two wavelengths, 360 and $269 \mathrm{~nm}$ (Fig.6), give $\mathrm{x}$ values for which $\mathrm{F}(\mathrm{x})$ is optimal. The three values of $\mathrm{x}$ equal to $\sim 0.35, \sim 0.5$ and $\sim 0.67$ correspond to the (DBMBF2) 2 :benzene, DBMBF2:benzene and DBMBF2:(benzene $)_{2}$ complexes, respectively.
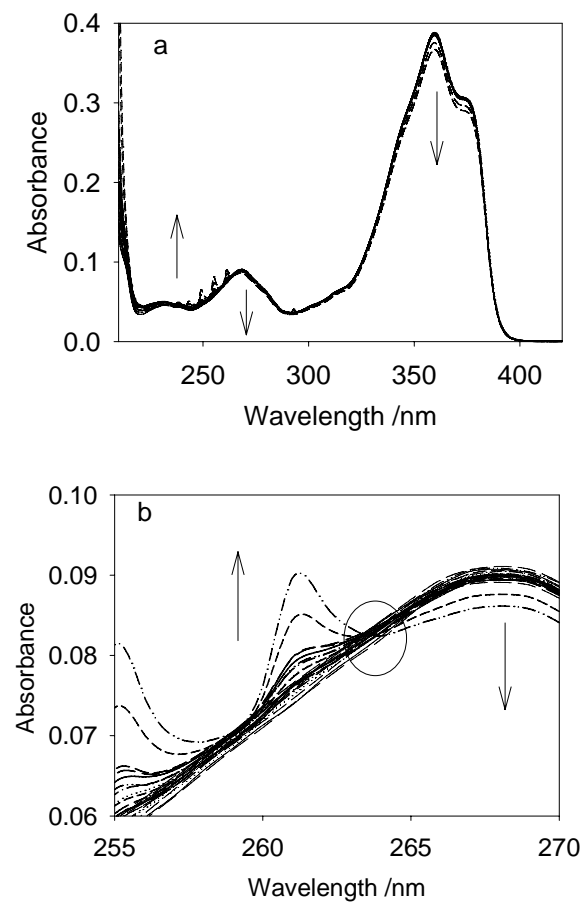

Fig.5 (a): Spectral evolution of DBMBF2 $10^{-5}$ mol. $\mathrm{L}^{-1}$ in cyclohexane upon addition of small aliquots of a mother solution of benzene $1.810^{-3}$ mol. $\mathrm{L}^{-1}$ in cyclohexane. Starting volume: $4 \mathrm{~cm}^{3}$, the concentration of benzene varies from $910^{-7}-1.2510^{-4} \mathrm{~mol} \cdot \mathrm{L}^{-1}$. (b): Zoom on the isobestic point at $264 \mathrm{~nm}, \operatorname{Abs}(264 \mathrm{~nm})=0.083 \pm 0.002$. 

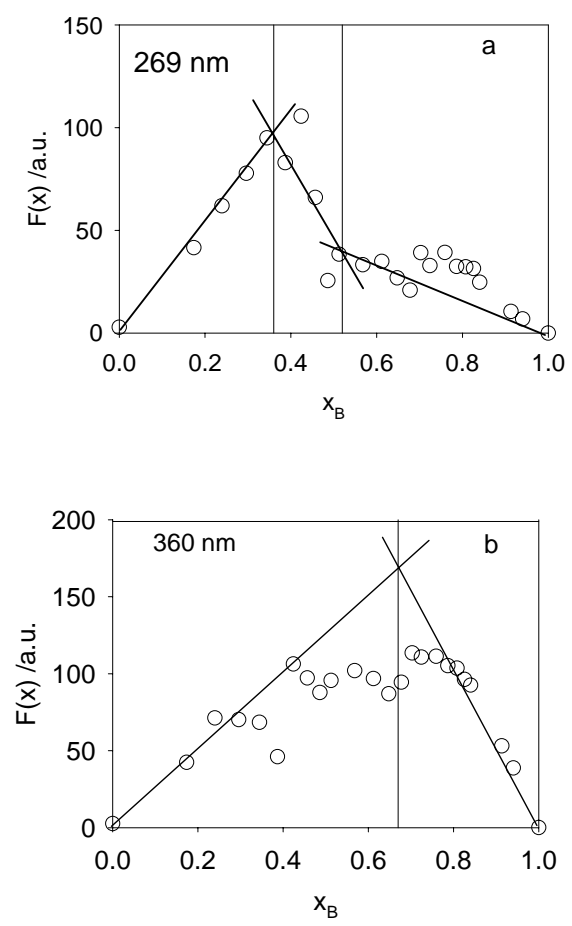

Fig.6 Job plots obtained from the titration of DBMBF2 $\left(10^{-5}\right.$ mol. $\left.\mathrm{L}^{-1}\right)$ with a mother solution of benzene $\left(1.810^{-3}\right.$ mol. $\left.\mathrm{L}^{-1}\right)$ in cyclohexane. Starting volume: $4 \mathrm{~cm}^{3}$, the concentration of benzene varies from $910^{-7}-1.2510^{-4} \mathrm{~mol} \cdot \mathrm{L}^{-1}$. $\mathrm{X}_{\mathrm{B}}$ is the benzene molar fraction.

To favor the formation of the complexes, we increase the DBMBF2 concentration by a factor 10. Figure 7 displays the spectral variation of DBMBF2 $10^{-4} \mathrm{~mol}^{-\mathrm{L}^{-1}}$ in cyclohexane upon addition of small aliquots of a solution of benzene $1.710^{-3} \mathrm{~mol} . \mathrm{L}^{-1}$ in cyclohexane. A decrease of the absorbance at $360 \mathrm{~nm}$ corresponding to DBMBF2 monomer and the presence of an isobestic point at $213 \mathrm{~nm}$ argue for the existence of an equilibrium at least between two absorbing species. The plots of Job diagrams at 269 and $360 \mathrm{~nm}$ (Fig.8), give $\mathrm{x}$ values, for which $\mathrm{F}(\mathrm{x})$ is optimal, equal to 0.5 and 0.66 . These values correspond to the DBMBF2:benzene and the DBMBF2:(benzene) $)_{2}$ respectively. 

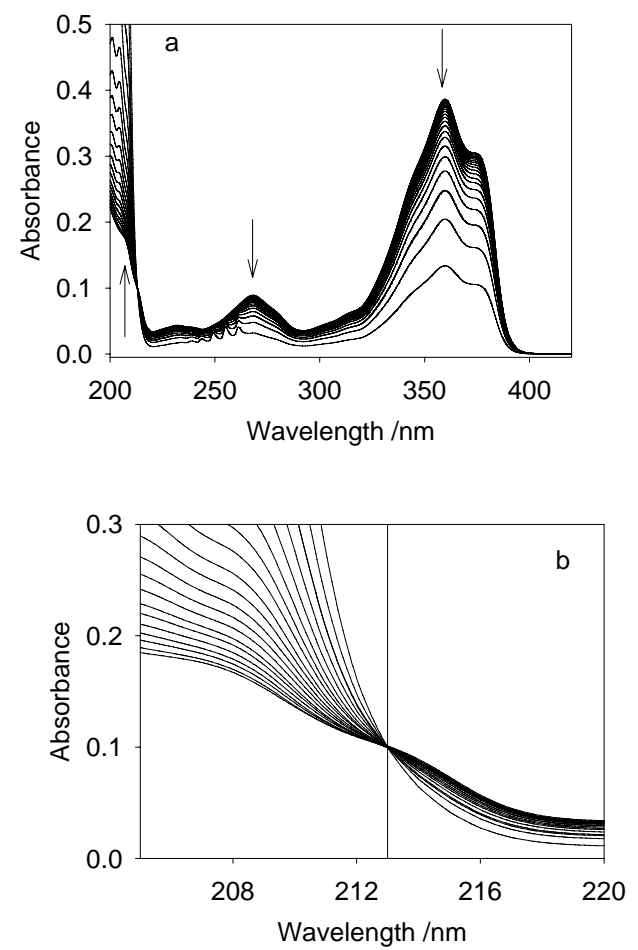

Fig.7 (a): Spectral evolution of DBMBF2 $10^{-4}$ mol. $\mathrm{L}^{-1}$ in cyclohexane upon addition of small aliquots of a mother solution of benzene $1.710^{-3} \mathrm{~mol} . \mathrm{L}^{-1}$ in cyclohexane. Starting volume: $4 \mathrm{~cm}^{3}$, the concentration of benzene varies from $8.710^{-6}-1.0910^{-3} \mathrm{~mol}^{-1} \mathrm{~L}^{-1}$. (b): Zoom on the isobestic point at $213 \mathrm{~nm}, \operatorname{Abs}(213 \mathrm{~nm})=0.100 \pm 0.002$. Optical pathlength: $1 \mathrm{~mm}$
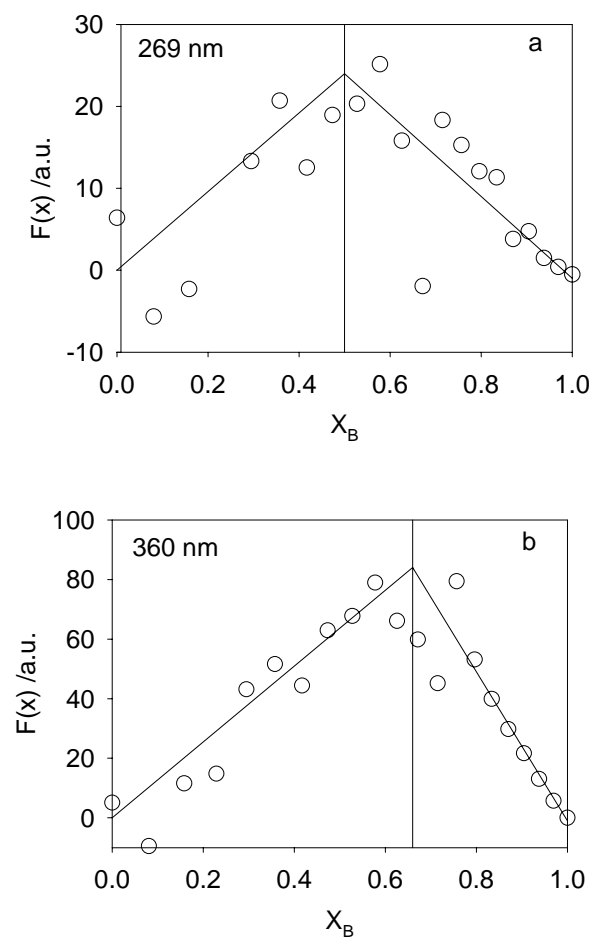

Fig.8 Job plots obtained from the titration of DBMBF2 $\left(10^{-4}\right.$ mol. $\left.\mathrm{L}^{-1}\right)$ with a mother solution of benzene $\left(1.710^{-3} \mathrm{~mol}^{-\mathrm{L}^{-1}}\right)$ in cyclohexane. Starting volume: $4 \mathrm{~cm}^{3}$, the concentration of benzene varies from $8.710^{-6}-1.0910^{-3} \mathrm{~mol} \cdot \mathrm{L}^{-1}$. $\mathrm{X}_{\mathrm{B}}$ is the benzene molar fraction. 
We found similar results when benzene is replaced by toluene (see Fig. 9). Here again, the spectral evolution in absorbance, displaying a clear isobestic point at $222 \mathrm{~nm}$, witnesses the existence of an equilibrium between the DBMBF2 monomer and a ground state complex. The Job diagrams plotted at 360 and $270 \mathrm{~nm}$ (Fig.10) clearly show the existence of three complexes, (DBMBF2) 2 :toluene, DBMBF2:toluene and DBMBF2:(toluene)2.
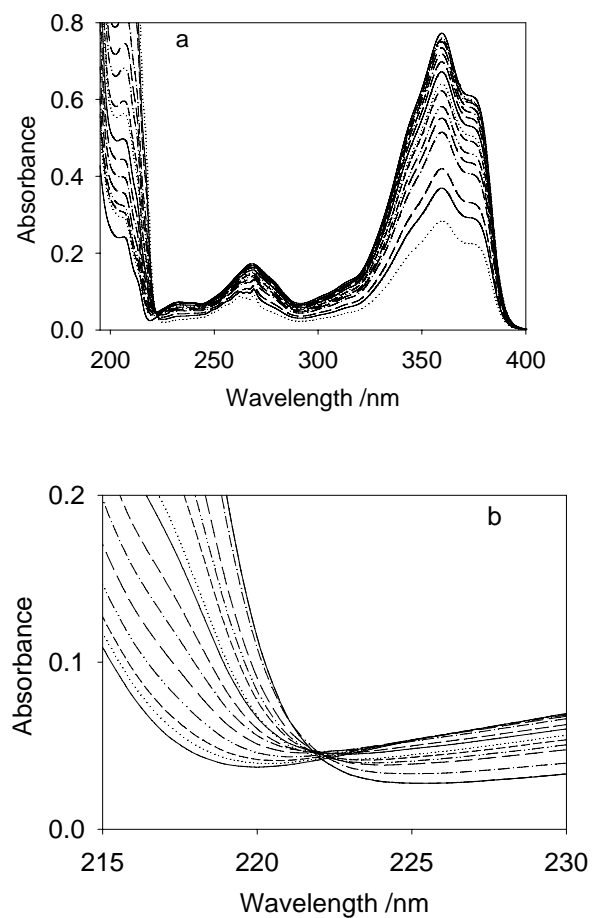

Fig.9 (a): Spectral evolution of DBMBF2 $10^{-4} \mathrm{~mol}^{-1} \mathrm{~L}^{-1}$ in cyclohexane upon addition of small aliquots of a solution of toluene $10^{-3}$ mol. $\mathrm{L}^{-1}$ in cyclohexane. Starting volume $4 \mathrm{~cm}^{3}$, the concentration of toluene varies from $2.510^{-6}-510^{-4} \mathrm{~mol} . \mathrm{L}^{-1}$. (b): Zoom on the isobestic point at $222 \mathrm{~nm}$. Abs(222 $\mathrm{nm})=0.045 \pm 0.005$. Optical pathlength: $2 \mathrm{~mm}$. 

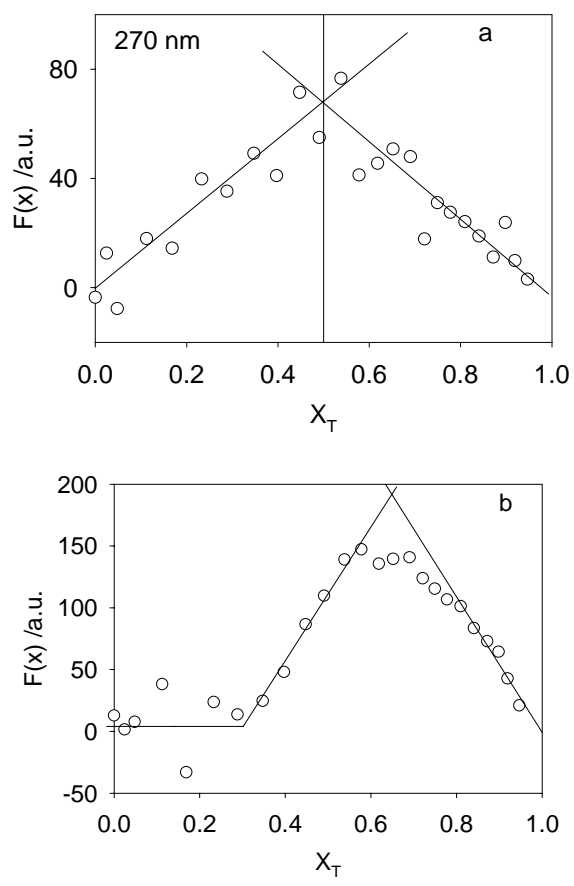

Fig.10 Job plots obtained from the titration of DBMBF2 $\left(10^{-4}\right.$ mol.L $\left.\mathrm{L}^{-1}\right)$ with toluene $\left(10^{-3}\right.$ mol.L $\left.\mathrm{L}^{-1}\right)$ in cyclohexane solutions. Starting volume $4 \mathrm{~cm}^{3}$, the concentration of toluene varies from $2.510^{-6}-$ $6.410^{-4} \mathrm{~mol} \cdot \mathrm{L}^{-1} . \mathrm{X}_{\mathrm{T}}$ is the toluene molar fraction.

\subsubsection{Fluorescence titrations}

These findings are corroborated with the fluorimetric titrations. Below $250 \mathrm{~nm}$, the intensity of the xenon lamp is very low and we could not excite the solutions at 213 and 222 $\mathrm{nm}$ for the titration of DBMBF2 with benzene and toluene, respectively. By exciting the mixtures of DBMBF2 and benzene in cyclohexane at $255 \mathrm{~nm}$, where the absorbances do not practically vary $(0.055 \pm 0.003)$, we can follow the equilibrium between the DBMBF2 monomer and the corresponding complex or complexes with benzene molecules. Note that at $255 \mathrm{~nm}$, the values of the extinction coefficient of DBMBF2 and benzene in cyclohexane are $5400 \pm 200$ and $205 \pm 10 \mathrm{~mol}^{-1} . \mathrm{L} \cdot \mathrm{cm}^{-1}$, respectively. Therefore, during the titration, the contribution of the benzene absorbance at $255 \mathrm{~nm}$ is negligible. 

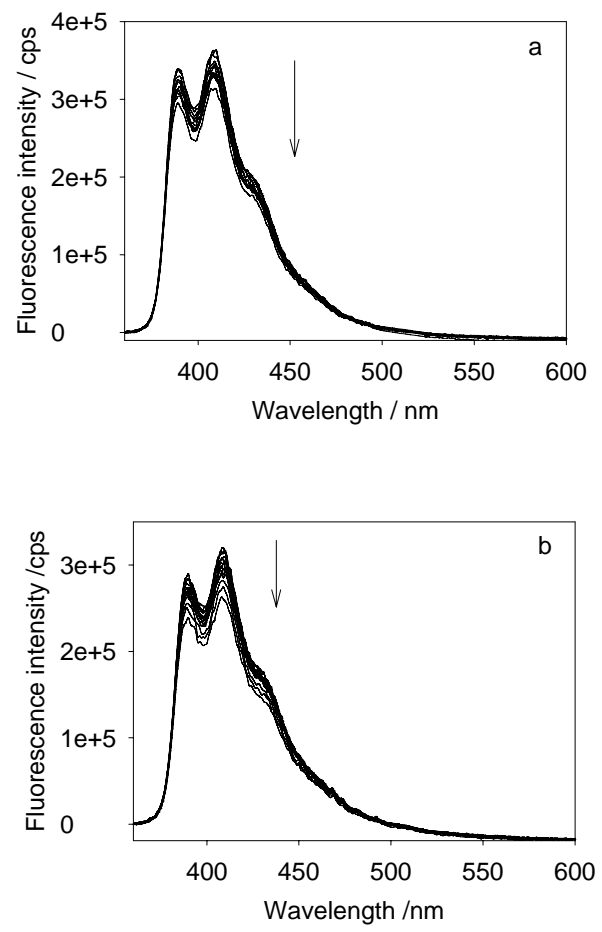

Fig.11 Evolution of the fluorescence intensity of DBMBF2 $10^{-4} \mathrm{~mol}^{-\mathrm{L}^{-1}}$ in cyclohexane upon addition of aliquots of a solution of benzene $1.710^{-3} \mathrm{~mol} . \mathrm{L}^{-1}$ (a) and of toluene $10^{-3} \mathrm{~mol} . \mathrm{L}^{-1}$ (b). The concentration of benzene varies from $8.710^{-6}$ to $2.6210^{-4} \mathrm{~mol} \cdot \mathrm{L}^{-1} \cdot \lambda_{\mathrm{exc}}=255 \mathrm{~nm}$ and the concentration of toluene varies from $510^{-6}$ to $2.3510^{-4} \mathrm{~mol} \cdot \mathrm{L}^{-1} \cdot \lambda_{\mathrm{exc}}=253 \mathrm{~nm}$

Figure 11 displays the change of the fluorescence intensity of the excited DBMBF2 $10^{-4}$ mol. $\mathrm{L}^{-1}$ in cyclohexane upon addition of small aliquots of a solution of benzene $1.710^{-3}$ mol. $\mathrm{L}^{-1}$ in the same solvent. In contrast to the results obtained with DBMBF2 in the binary mixtures of cyclohexane and benzene, the position of the maximum of the fluorescence band remains unchanged and the intensity of fluorescence decreases continuously upon addition of benzene. This result strongly supports the hypothesis of the formation of ground state complexes which are non fluorescent in the excited state. We use the Job method extended to fluorescence titration [7] to determine the stoichiometry of the complexes: the fluorescence area is plotted as a function of the number of benzene equivalent, $n$, $(\mathrm{n}=[$ benzene]/[DBMBF2]) (Fig.12). The curve displays two linear regimes which intersect at a value equal to unity, which corresponds to the 1:1 stoichiometry of the complex. One can also note that even at high concentration ratio [benzene]/[DBMBF2], the total fluorescence area still decreases and no extra fluorescence band which could be attributed to the presence of any exciplex is detected over $360-750 \mathrm{~nm}$.

The same results can be obtained with toluene when the solutions of DBMBF2 in cyclohexane are excited at $253 \mathrm{~nm}$, where the variation of absorbance is weak $(0.050 \pm 0.003)$ (Fig.11). Here again, at $253 \mathrm{~nm}$, the value of the extinction coefficient of toluene $(170 \pm 10)$ is negligible when compared with that of DBMBF2 $\left(4900 \pm 200 \mathrm{M}^{-1} \mathrm{~cm}^{-1}\right)$. The position of the maximum of the fluorescence band remains unchanged and the fluorescence intensity of excited DBMBF2 decreases continuously upon addition of small aliquots of toluene. Here again, the plot of the total fluorescence area as a function of the ratio of concentrations [toluene]/[DBMBF2] gives two linear regimes which intersect at a value equal to 1 (Fig.12). 
This value indicates the existence of a complex of stoichiometry 1:1 which is not fluorescent or whose very weak fluorescence cannot be detected.
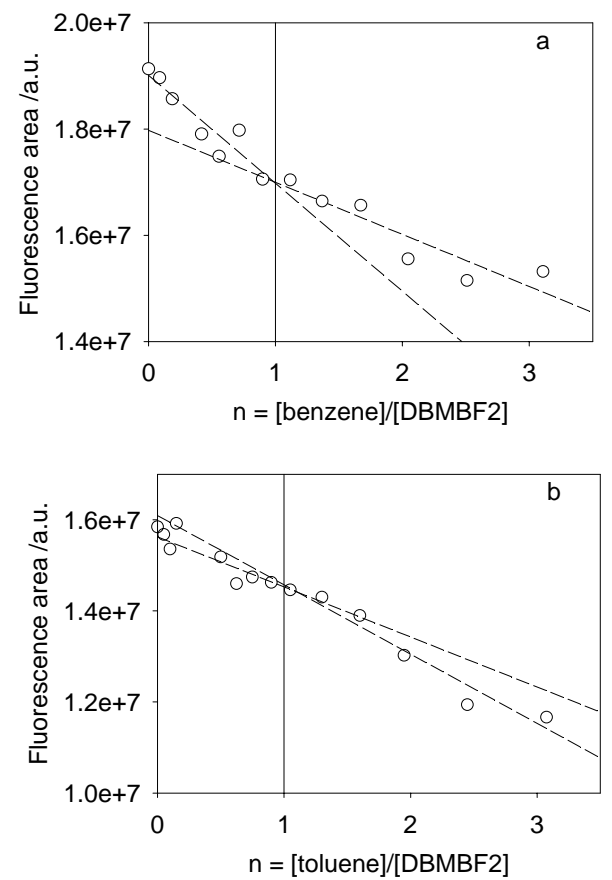

Fig.12 Plot of fluorescence area as a function of the number of aromatic per DBMBF2 molecule for benzene (a) and toluene (b). The fluorescence areas of mixtures are normalized to the average absorbance value at $255 \mathrm{~nm}$ for benzene $(0.055 \pm 0.003)$ and $253 \mathrm{~nm}$ for toluene $(0.050 \pm 0.003)$.

For benzene, $\mathrm{n}$ varying from 0.08 to 3.11 corresponds to a benzene concentration varying from $8.710^{-6}$ to $2.6210^{-4} \mathrm{~mol} . \mathrm{L}^{-1}$. For toluene, $\mathrm{n}$ varying from 0.05 to 3.08 corresponds to a toluene concentration varying from $510^{-6}$ to $2.3510^{-4}$ mol. $\mathrm{L}^{-1}$.

When analyzing the decay of the fluorescence intensity or area as a function of the quencher concentration [Q] using the Stern-Volmer equation [23], we assume the existence of a static quenching of DBMBF2 due to the formation of 1:1 ground state CT complexe, DBMBF2benzene.

DBMBF2 $+\mathrm{Q} \Leftrightarrow$ DBMBF2:Q with the equilibrium constant $\mathrm{K}_{\text {ass }}$

Such a model leads to the following equation

$\frac{I_{0}}{I}=1+K_{S V}[Q]$

where $I_{0}$ and $I$ are the fluorescence intensities in absence and in the presence of the quencher, respectively, $K_{s v}$, which is the Stern-Volmer constant, corresponds to the association constant of the complex for a static quenching, and [Q], the quencher concentration.

The good fit of the experimental data of fluorescence titrations (Fig. 11) with equation 6 argues for the accuracy of the chosen model (see Fig. 13). This allows to extract the values of the association constant for the 1:1 complex DBMBF2:Q for the two mixtures with benzene and toluene. 

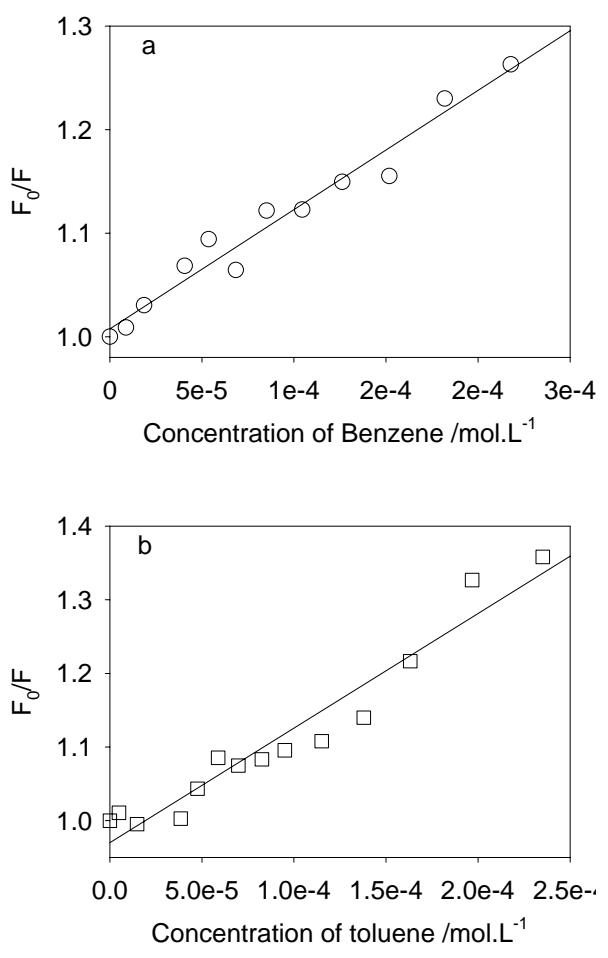

Fig.13 Stern-Volmer plots from the variation of the fluorescence intensity of DBMBF2 in cyclohexane upon addition of small aliquots of $(\mathrm{O})$ benzene (a) and ( $\square$ ) toluene (b) - Fit of the experimental data of Fig. 11 with equation 6, taking into account the change of the DBMBF2 concentration.

For benzene,

$\mathrm{K}_{\mathrm{SV}}=\mathrm{K}_{\mathrm{ass}}(\mathrm{DBMBF} 2$ :benzene $)=1150 \pm 100 \mathrm{~L} \cdot \mathrm{mol}^{-1}$

and for toluene,

$\mathrm{K}_{\mathrm{SV}}^{\prime}=\mathrm{K}^{\prime}{ }_{\text {ass }}(\mathrm{DBMBF} 2:$ toluene $)=1550 \pm 200 \mathrm{~L} \cdot \mathrm{mol}^{-1}$

Our data indicates a better association of DBMBF2 with toluene than with benzene, which can be explained in terms of a higher electron donor ability of toluene as compared to benzene. One can also note that the values of the present association constants for the 1:1 complex are much higher than those determined by Valat et al. from the deconvolution of the fluorescence spectra of DBMBF2 in binary mixtures of cyclohexane with benzene or with toluene, based on the assumption of the existence of two exciplexes DBMBF2:benzene and DBMBF2:(benzene) ${ }_{2}[3]$. They found values of the association constant in the excited state equal to 2.7 and $14 \mathrm{~L}_{\mathrm{mol}}{ }^{-1}$, for benzene and toluene respectively [3].

We noted that the model proposed by Chow et al. cannot explain all the data collected from many of their studies. For instance, the authors had recorded the fluorescence lifetime of DBMBF2 monomer $\left(10^{-5} \mathrm{~mol} . \mathrm{L}^{-1}\right)$ in acetonitrile in the absence and in the presence of benzene. They found that the lifetime of excited DBMBF2, equal to $340 \mathrm{ps}$ in pure acetonitrile, decreases to less than 30 ps (limit of their detection) in the presence of low 
concentrations of benzene $\left(\sim 0.1 \mathrm{~mol} . \mathrm{L}^{-1}\right)$ and starts to increase above $1 \mathrm{~mol} . \mathrm{L}^{-1}$ up to 0.65 , $1.15,1.75 \mathrm{~ns}$, in mixtures where benzene and acetonitrile become co-solvents with benzene concentrations equal to $1.02,3.76$ and $7.78 \mathrm{~mol} . \mathrm{L}^{-1}$, respectively [5]. In pure benzene, the lifetime of the complex is $2.1 \mathrm{~ns}$ [5]. The same behavior was observed with toluene and xylene, but 3 and $6 \mathrm{~mol} . \mathrm{L}^{-1}$, respectively, were needed to induce the same lifetime increase [5]. Applying the model of formation of exciplexes does not allow to explain why the lifetime of DBMBF2 first decreases and then increases upon addition of the aromatic molecules. Furthermore, it does not explain why the formation of the 1:1 and 1:2 charge transfer exciplexes would be more favorable for benzene than for its methylated derivatives which display higher electron donating ability.

Our present results and the proposed model allow to explain all the results of Chow's group:

- The decrease of the DBMBF2 excited state lifetime (less than $30 \mathrm{ps}$ ) would be due to the formation in the ground state of charge transfer complexes of low stoichiometry, which decay preferentially via non radiative pathways when they are excited. These are the 2:1, 1:1 and 1:2 ground state CT complexes solvated by acetonitrile or cyclohexane molecules, whose existence is demonstrated in the present work. Their association constant increases with the electron donating ability of the aromatic molecule.

- The increase of the fluorescence lifetime corresponds to the presence of new ground state charge transfer complexes of DBMBF2 with the aromatics with high stoichiometry, DBMBF2:(aromatic) $)_{n}$ whose excited state have long lifetime and are highly fluorescent. The nature of these new ground state CT complexes and the reason why their formation seems to be less favorable with the methylation of the aromatic compound is now discussed.

\subsubsection{Nature of the high stoichiometry complex}

From our present findings, we know that very high concentration of the aromatic compound is needed to form the high stoichiometry ground state complex: i.e. $\sim 0.7$ mol.L $\mathrm{L}^{-1}$ of benzene in cyclohexane for $10^{-5}$ mol.L $\mathrm{L}^{-1}$ of DBMBF2 is needed for the formation of the complex. On the other hand, it is well-known from the literature data that benzene molecules in cyclohexane start to aggregate at similar concentration [24] and that the aggregates, in their excited state, display a wide and structureless fluorescence band extending from 265 to 360 $\mathrm{nm}$ [25-27]. These considerations bring us to suggest that the interaction of benzene aggregates with DBMBF2 molecules could lead to the formation of stable charge transfer complexes in the ground state. The electron affinity of clusters of benzene could be very different from that of the isolated benzene and therefore the charge transfer character of the high stoichiometry complex could be enhanced. This assumption is based on the large Stokes shift value, $3800 \mathrm{~cm}^{-1}$, which is usually found for complexes with high CT character. In their excited state, these complexes seem to decay preferentially via a radiative decay pathway. The hypothesis of the participation of aggregates in the formation of the high stoichiometry ground state complex is strengthened by the fact that for toluene and xylene, very high concentrations, 3 and $6 \mathrm{~mol} . \mathrm{L}^{-1}$, respectively, were needed for the observation of the fluorescent complex as compared to $1 \mathrm{~mol} . \mathrm{L}^{-1}$ for benzene [5]. Because of the presence of the methyl group, toluene and xylene aggregates are less easily formed than benzene ones. Moreover, the formation of these aggregates should be easier in a non-interacting co-solvent such as cyclohexane, than in acetonitrile. On the other hand, the complex formed between DBMBF2 and toluene aggregates displays a stronger CT character than the complex with benzene aggregates, as witnessed by the large value of the Stokes shift, $4520 \mathrm{~cm}^{-1}$ for toluene as compared to $3800 \mathrm{~cm}^{-1}$ for benzene (calculated from Table 1) observed for this system. 
The knowledge of the lifetime of the singlet excited DBMBF2 in the binary mixtures could also bring some clues on the nature of the complexes. In their previous works, Chow et al. found, that:

1. in cyclohexane solutions, above $0.3 \mathrm{~mol} . \mathrm{L}^{-1}$ of benzene, the fluorescence lifetime attributed to the exciplex regularly increases with increased benzene concentration: $\tau_{\mathrm{s}}$ $=0.35 \mathrm{~ns}\left([\mathrm{~B}]=0.346 \mathrm{~mol} . \mathrm{L}^{-1}\right), 0.40 \mathrm{~ns}\left(0.602 \mathrm{~mol} . \mathrm{L}^{-1}\right), 0.52 \mathrm{~ns}\left(0.994 \mathrm{~mol} . \mathrm{L}^{-1}\right)$ and $0.63 \mathrm{~ns}\left(1.410 \mathrm{~mol} . \mathrm{L}^{-1}\right), 0.71 \mathrm{~ns}\left(1.824 \mathrm{~mol} . \mathrm{L}^{-1}\right)$ and $2.1 \mathrm{~ns}$ in pure benzene $(11.2$ mol. $\left.\mathrm{L}^{-1}\right)$ [5]. The same phenomenon was observed with toluene: $\tau_{\mathrm{s}}=1.0 \mathrm{~ns}([\mathrm{~T}]=$ 0.273 mol. $\left.\mathrm{L}^{-1}\right), 1.3 \mathrm{~ns}\left(0.716 \mathrm{~mol}^{-\mathrm{L}^{-1}}\right), 1.8 \mathrm{~ns}\left(1.335 \mathrm{~mol} . \mathrm{L}^{-1}\right), 2.5 \mathrm{~ns}\left(2.887 \mathrm{~mol}^{-1} \mathrm{~L}^{-1}\right)$, $3.2 \mathrm{~ns}\left(5.709 \mathrm{~mol}^{-\mathrm{L}^{-1}}\right)$ and $3.7 \mathrm{~ns}$ in pure toluene [2].

2. in acetonitrile solutions, that above $1 \mathrm{~mol} . \mathrm{L}^{-1}$, the fluorescence lifetime that they attributed to the exciplex regularly increases with increased benzene concentration: $\tau_{\mathrm{s}}$ $=0.65 \mathrm{~ns}\left([\mathrm{~B}]=1.02 \mathrm{~mol} . \mathrm{L}^{-1}\right), 1.15 \mathrm{~ns}\left(3.76 \mathrm{~mol} . \mathrm{L}^{-1}\right), 1.75 \mathrm{~ns}\left(7.78 \mathrm{~mol} . \mathrm{L}^{-1}\right)$ and 2.1 ns in pure benzene [5]. The same phenomenon was observed with toluene above a concentration of $3 \mathrm{~mol} . \mathrm{L}^{-1}: \tau_{\mathrm{s}}=3.5 \mathrm{~ns}\left([\mathrm{~T}]=4.67 \mathrm{~mol} . \mathrm{L}^{-1}\right), 6.2 \mathrm{~ns}\left(6.17 \mathrm{~mol} . \mathrm{L}^{-1}\right), 7.3$ ns $\left(7.8\right.$ mol. $\left.\mathrm{L}^{-1}\right)$ but $4.1 \mathrm{~ns}$ in pure toluene [5].

These variations can be explained with our proposed model if the CT complex were more polar in the excited state than in the ground state. Harju et al., by studying the solvent viscosity and polarity effects on the picosecond barrier crossing reaction of DBMBF2 in nnitrile and n-alcohols, had shown that the transition state of DBMBF2 monomer itself is highly polar with respect to the fluorescent state and that the subsequent solvation effects contribute to the reaction rates [29]. In the present case, if the CT complex were more polar in the excited state, the lifetime of the excited complex would strongly depend on the polarity of the solvent: the more polar would be the mixture, the longer would be the lifetime of the excited state due to its stabilisation via solvation of the charges. Because acetonitrile (dielectric constant $\left.\varepsilon_{\mathrm{S}}=35.94\right)$ is much more polar than cyclohexane $\left(\varepsilon_{\mathrm{S}}=2.02\right)$ and benzene $\left(\varepsilon_{S}=2.27\right)$ or toluene $\left(\varepsilon_{S}=2.38\right)$ [28], the lifetimes of the CT complex measured in binary mixtures of acetonitrile and benzene (or toluene) are longer than the ones found in binary mixtures of cyclohexane and benzene (or toluene). For the same reason, the lifetime of the excited CT complex in pure toluene $(4.1 \mathrm{~ns})$ is shorter than in the mixtures with acetonitrile (7.3 ns).

The difference of properties observed for the high stoichiometry complexes as compared to the low stoichiometry ones must arise from the nature of the interactions between DBMBF2 with individual or aggregates of aromatic molecules. To understand the nature of the interactions and predict the sites of interaction between Benzene and DBMBF2, we determine the structure of the 1:1 complex using semi-empirical quantum chemistry calculations. At the present stage, it is not possible to perform calculations with a large number of aromatic molecules and we therefore restrict our study to the low stoichiometry complex, DBMBF2:benzene.

\subsubsection{Structures of the 1:1 complex of DBMBF2:Benzene}

For the isolated DBMBF2, a single structure was found with the two aromatic cycles symmetrical along the axis joining the middle cycle to the boron atom and the BF2 group orthogonal to the middle cycle (Fig.14). This symmetrical structure corresponds to the one determined via X-rays diffraction [10]. 


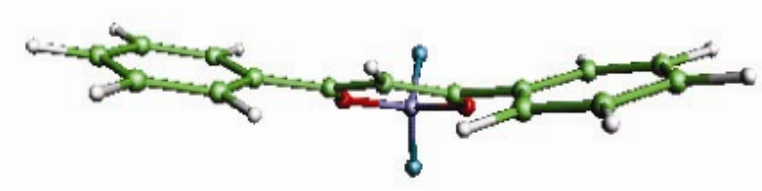

Fig 14 structure of the isolated DBMBF2

Many structures of the 1:1 complex of DBMBF2 and benzene were found. Among the different configurations, we select the three configurations which display the lowest potential energies, the other ones lying at significantly higher energy. They are very close in energy: $4.48,-4.60$ and $-4.73 \mathrm{kcal}^{\mathrm{mol}}{ }^{-1}$. Figure 15 displays the three corresponding sites of interaction between the two moieties. For all structures, the dispersion interaction contributes the most in the stabilisation of the complex, while the electrostatic interaction appears to be the highest when the benzene ring is proximate and perpendicular to the phenyl ring of DBMBF2. Table 3 displays the contributions of the electrostatic, dispersion, repulsion and polarization in the total energy of the complex.

Table 3: Different contributions in the total energy of the 1:1 complex

\begin{tabular}{|c|c|c|c|}
\hline Complexe DBMBF2:Benzene & 1 & 2 & 3 \\
\hline \hline Interaction between DBMBF2 and Benzene $/ \mathrm{kcal} \mathrm{mol}^{-1}$ & & & \\
\hline Electrostatic & -1.99 & -1.11 & -1.35 \\
Dispersion & -4.73 & -5.76 & -5.37 \\
Repulsion & 2.63 & 2.66 & 2.71 \\
\hline Total & -4.09 & -4.21 & -4.01 \\
\hline Polarization $/ \mathrm{kcal} \mathrm{mol}^{-1}$ & -0.64 & -0.39 & -0.47 \\
\hline \hline Total Energy $/ \mathrm{kcal}^{-1}$ & -4.73 & -4.60 & -4.48 \\
\hline
\end{tabular}

The present calculations bring many informations which support our findings for the low stoichiometry complexes:

- many sites of interaction between DBMBF2 and benzene molecules are available

- the 1:1 complex can display three different and stable structures and therefore could be easily formed in solution

- for the 1:2 stoichiometry, among the numerous possible structures, two symmetrical structures would be available. With a first complex having two symmetrical phenyl rings, one can expect a symmetrical interaction of benzene molecules on both phenyl rings of DBMBF2. The second complex would have each benzene ring facing the DBMBF2 phenyl ring in a tilted position. 

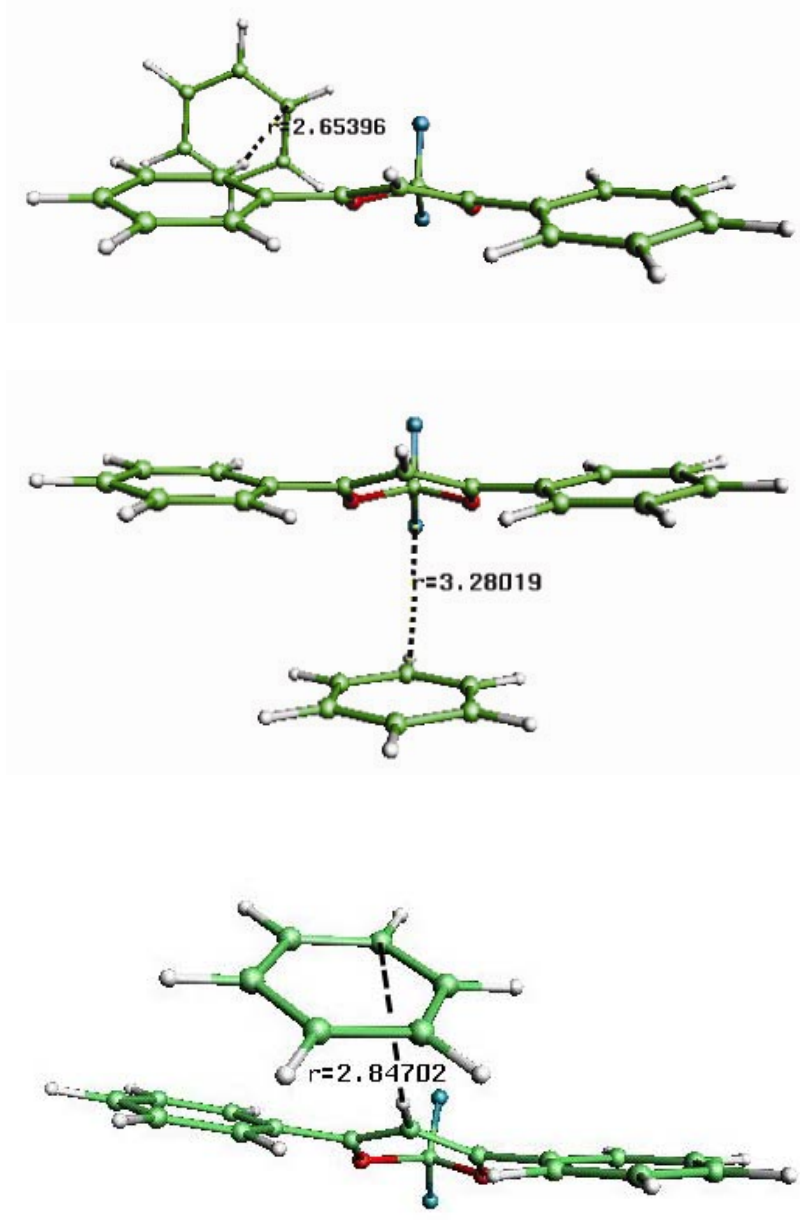

Fig 15 Structures of the most stable configurations of DBMBF2:benzene complex

These low stoichiometry complexes are formed in the ground state and their excited states decay via non radiative pathways.

More complex calculations on the high stoichiometry complexes, involving an organized aggregate of benzene, would undoubtedly bring a better understanding of the properties of these complexes, in particular, the nature of their transitions. This implies many steps including

- the study of the aggregates aromatic molecules (3, 4 or more) and the search of the most stable conformations

- the study of the interaction of DBMBF2 with the aggregates

- the calculations of their transitions

Such complete and time consuming calculations could not be done during the process of this study.

\section{Conclusion}

In previous work, it was found that (dibenzoylmethanato)boron difluoride (DBMBF2) was able to form with benzene (B) and its methylated derivatives (MB) in solution strongly 
fluorescing complexes. These were thought to be the 1:1 and 1:2 exciplexes, complexes of the singlet excited state of DBMBF2 with one or two B or MB molecules. The present work sheds new light on the nature of the various complexes and also allows the explanation of the previously observed unexpected evolution of the DBMBF2 lifetime with the aromatic concentration.

Two types of complexes can be formed, both in the ground state, in cyclohexane solution. The first type has low stoichiometry and contains one or two aromatic compounds. Their association constants are 1150 and $1550 \mathrm{~L}^{-\mathrm{mol}^{-1}}$ for the 1:1 complex for benzene and toluene, respectively. Upon excitation, these complexes do not fluoresce and their excited states decay very rapidly via non-radiative pathways. These results allow us to explain why the lifetime of excited DBMBF2, measured in previous works, initially quickly decreases with low concentrations of the aromatic compound. They also allow us to explain the reactivity of DBMBF2 in the porous matrices exposed to gas mixture containing traces of benzene or toluene. We also obtain the formation of the non fluorescent 1:1 CT complex of DBMBF2 with benzene or toluene. The fluorescence of DBMBF2 monomer is therefore quenched when the sensor is exposed to the pollutants.

The second type of complex, with high stoichiometry, corresponds to a ground state CT complex between DBMBF2 and an aggregate of the aromatic compound. In contrast to the first type of complex, the high stoichiometry CT complex upon excitation displays a strong fluorescence and a long lifetime. The CT character which we have attributed to the complex is indicated by its large Stokes shift value $\left(3800\right.$ and $4250 \mathrm{~cm}^{-1}$ for benzene and toluene, respectively). Because of the enhanced polarity of the CT complex in the excited state, the species is stabilized in increasing polar media. As a consequence, its lifetime strongly varies with the polarity of the binary mixtures.

\section{Acknowledgements}

T.-T. Truong acknowledges the support of the Commissariat à l'Energie Atomique and the Agence de l'Environnement et de la Maîtrise de l'Energie (CEA/ADEME, training grant $\mathrm{N}^{\circ}$ BOU0060). 


\section{References}

1. M.-L. Calvo-Muñoz,, T.-T. Truong and T.-T. Tran-Thi, Chemical sensors of monocyclic aromatic hydrocarbons based on sol-gel materials: kinetics of trapping of the pollutants and sensitivity of the sensor, Sensors \& Actuators B, 2002, 87, 173-183.

2. Y. L. Chow, C. J. Johansson, Exciplexes binding energy and kinetic rate constants of the interaction between singlet excited state (Dibenzoylmethanato)boron difluoride and substituted benzenes: J. Phys. Chem., 1995, 99, 17566-17572.

3. P. Valat, V. Wintgens, Y. L. Chow, J. Kossanyi, Unusually strong emission from an exciplex formed between benzenoid solvents and dibenzoylmethanatoboron difluoride. Formation of a triplex, Can. J. Chem., 1995, 73, 1902-1913.

4. Y. L. Chow, X. Cheng, C. I. Johansson, Molecular interactions of dibenzoylmethanatoboron difluoride in the excited and ground states in solution, $J$. Photochem. Photobiol. A: Chem., 1991, 57, 247-255.

5. Y. L. Chow, S.-S. Wang, S. Z.-L. Liu, V. Wintgens, P. Valat, J. Kossanyi, Photochemistry and photophysics of dibenzoylmethanatoboron difluoride with cyclic dienes, New J. Chem., 1994, 18, 923-936.

6. P. Job, Formation and stability of inorganic complexes in solution, Ann. di Chim. Appl., 1928, 9, 113-199. Z. D. Hill, P. MacCarthy, Novel approach to Job's method. An undergraduate experiment., J. Chem. Edu., 1986, 63, 162-167.

7. J.-F. Lipskier, T.-H. Tran-Thi, Supramolecular assemblies of porphyrins and phtalocyanines bearing oppositely charged substituents. First evidence of heterotrimer formation, Inorg. Chem., 1993, 32, 722-731.

8. N. M. D. Brown, P. Bladon, Spectroscopy and structure of (1,3-diketonato)boron difluorides and related compounds, J. Chem. Soc. A, 1969, 69, 526-532.

9. R. A. Velapoldi, K. D. Mielenz, Standard Reference Materials: A Fluorescence Standard Reference Material Quinine sulfate Dihydrate, National Bureau of Standards, U.S. Department of Commerce, 1980, pp 260-264.

10. A. G. Mirochnik, B. V. Bukvetskii, E. V. Gukhman, P. A. Zhikhareva, V. E. Karasev, Crystal structure and excimer fluorescence of dibenzoylmethanatoboron difluoride, Russian Chem. Bull., 2001, 50, 1612-1615.

11. M. J. S. Dewar, E. G. Zoebisch, E. F. Healy, J. J. P. Stewart, AM1: A New General Purpose Quantum Mechanical Molecular Model, J. Am. Chem. Soc., 1985,107, 39023909.

12. W. J. Hehre, J. A. Pople, Self-consistent molecular orbital methods. IX. An extended Gaussian-type basis for molecular orbital studies of organic, J. Chem. Phys., 1971, 54, 724-728. P. C. Hariharan, J. A. Pople, The influence of polarization functions on MO hydrogenation energies, Theor. Chim. Acta, 1973, 28, 213-222.

13. M. J. Frisch, G. W. Trucks, H. B. Schlegel, G. E. Scuseria, M. A. Robb, J. R. Cheeseman, V. G. Zakrzewski, J. A. Montgomery, Jr., R. E. Stratmann, J. C. Burant, S. Dapprich, J. M. Millam, A. D. Daniels, K. N. Kudin, M. C. Strain, O. Farkas, J. Tomasi, V. Barone, M. Cossi, R. Cammi, B. Mennucci, C. Pomelli, C. Adamo, S. Clifford, J. Ochterski, G. A. Petersson, P. Y. Ayala, Q. Cui, K. Morokuma, N. Rega, P. Salvador, J. J. Dannenberg, D. K. Malick, A. D. Rabuck, K. Raghavachari, J. B. Foresman, J. Cioslowski, J. V. Ortiz, A. G. Baboul, B. B. Stefanov, G. Liu, A. 
Liashenko, P. Piskorz, I. Komaromi, R. Gomperts, R. L. Martin, D. J. Fox, T. Keith, M. A. Al-Laham, C. Y. Peng, A. Nanayakkara, M. Challacombe, P. M. W. Gill, B. Johnson, W. Chen, M. W. Wong, J. L. Andres, C. Gonzalez, M. Head-Gordon, E. S. Replogle and J. A. Pople, GAUSSIAN 98, revision A7, GAUSSIAN, Inc., Pittsburgh PA, 2002.

14. T. H. Jr. Dunning, Gaussian basis sets for use in correlated molecular calculations. I. The atoms boron through neon and hydrogen, J. Chem. Phys., 1989, 90, 1007-1023.

15. JAGUAR 4.1, SCHRÖDINGER, Inc: Portland, OR, 1991-2001

16. R. Krishnan, J. S. Binkley, R. Seeger and J. A. Pople, Self-consistent molecular orbital methods. XX. A basis set for correlated wave functions, J. Chem. Phys., 1980, 72, 650-654.

17. F. Vignie-Maeder, P. Claverie, The exact multicenter multipolar part of a molecular charge distribution and its simplified representations, J. Chem. Phys., 1988, 88, 49344948.

18. V. Brenner, Modélisation des forces intermoléculaires et caractérisation des hypersurfaces d'énergie potentielle: applications à de petits agrégats de Van der Waals, Thèse de l'Université de Paris-Sud, nº d'ordre: 2849, 1993.

19. S. Kirkpatrick, C. D. Gelatt, M. P. Vecchi, Optimisation by simulated annealing, Science, 1983, 220, 671-680. S. Kirkpatrick, Optimization by simulated annealing: Quantitative studies, J. Stat. Phys., 1984, 34, 975-986.

20. N. Metropolis, A. W. Rosentbluth, M. N. Rosenbluth, A. H. Teller, E. Teller, Equations of state calculations by fast computing machine, J. Chem. Phys., 1953, 21, 1087-1092.

21. W. H. Press, B. P. Flannery, S. A. Teukolsky, W. T. Vetterling - Numerical Recipes: The Art of Scientific Computing - Cambridge, UK, Cambridge University Press (1988).

22. Y. L. Chow, C. I. Johansson, Z.-L. Liu, Ground and excited-state electron donoracceptor (EDA) complexes from (dibenzoylmethanato)boron difluoride and substituted benzenes: their relation to the reaction mechanism, J. Phys. Chem.., 1996, 100, 13381-13385.

23. O. Stern, M. Volmer, The extinction period of fluorescence, Phys. Z., 1919, 20, 183 188; See also J. R. Lakowicz in Principles of fluorescence spectroscopy, Plenum Press, New York, 1983.

24. P. H. Bell; W. P. Davey, An X-Ray study of liquid benzene, cyclohexane and their mixtures, J. Chem. Phys., 1941, 9, 441-450.

25. I. B. Berlman in Handbook of fluorescence spectra of aromatic molecules, ed. I.B. Berlman, Academic Press, New York, $2^{\text {nd }}$ edn, (1971)

26. T. V. Ivanova, G. A. Mokeeva, B. Ya Sveshnikov, Optics \& Spectrosc., 1961, 12, 325-328.

27. J. B. Birks, C. L. Braga, M. D. Lumb, Excimer fluorescence. VI. Benzene, toluene, pxylene and mesitylene, Proc. Roy. Soc.A, 1965, 283, 83-99.

28. J. A. Riddick, W. B. Bunger, T. K. Sakano, Techniques of Chemistry. Vol.II. Organic solvents. Physical properties and methods of purification, Fourth edition, John Wiley \& Sons, New York, 1986. 
29. T. O. Harju, J. E. I. Korppi-Tommola, A. Herbert Huitzer, C. A. G. O. Varma, Barrier crossing reaction of electronically excited DBMBF2 in n-nitriles: the role of solvent polarity on activation energy, J. Phys. Chem., 1996, 100, 3592-3600. 